Delft University of Technology

\title{
Transient phase fraction and dislocation density estimation from in-situ X-ray diffraction data with a low signal-to-noise ratio using a Bayesian approach to the Rietveld analysis
}

Wiessner, Manfred; Angerer, Paul; van der Zwaag, Sybrand; Gamsjäger, Ernst

DOI

10.1016/j.matchar.2020.110860

Publication date

2021

Document Version

Final published version

Published in

Materials Characterization

\section{Citation (APA)}

Wiessner, M., Angerer, P., van der Zwaag, S., \& Gamsjäger, E. (2021). Transient phase fraction and dislocation density estimation from in-situ X-ray diffraction data with a low signal-to-noise ratio using a Bayesian approach to the Rietveld analysis. Materials Characterization, 172, [110860].

https://doi.org/10.1016/j.matchar.2020.110860

Important note

To cite this publication, please use the final published version (if applicable).

Please check the document version above.

\footnotetext{
Copyright

Other than for strictly personal use, it is not permitted to download, forward or distribute the text or part of it, without the consent of the author(s) and/or copyright holder(s), unless the work is under an open content license such as Creative Commons.

Takedown policy

Please contact us and provide details if you believe this document breaches copyrights.

We will remove access to the work immediately and investigate your claim.
} 
Green Open Access added to TU Delft Institutional Repository

'You share, we take care!' - Taverne project

https://www.openaccess.nl/en/you-share-we-take-care

Otherwise as indicated in the copyright section: the publisher is the copyright holder of this work and the author uses the Dutch legislation to make this work public. 


\title{
Transient phase fraction and dislocation density estimation from in-situ $\mathrm{X}$-ray diffraction data with a low signal-to-noise ratio using a Bayesian approach to the Rietveld analysis
}

\author{
Manfred Wiessner ${ }^{\mathrm{a}, *}$, Paul Angerer ${ }^{\mathrm{b}}$, Sybrand van der Zwaag ${ }^{\mathrm{c}}$, Ernst Gamsjäger ${ }^{\mathrm{d}}$ \\ ${ }^{a}$ Anton Paar GmbH, Anton-Paar-Str. 20, 8054 Graz, Austria \\ ${ }^{\mathrm{b}}$ Materials Center Leoben Forschung GmbH, Roseggerstr. 12, 8700 Leoben, Austria \\ ${ }^{\mathrm{c}}$ Novel Aerospace Materials group. Faculty of Aerospace Engineering, Delft University of Technology, Kluyverweg 1, 2629 HS Delft, The Netherlands \\ ${ }^{\mathrm{d}}$ Institute of Mechanics, Montanuniversität Leoben, Franz-Josef-Str. 18, 8700 Leoben, Austria
}

\section{A R T I C L E I N F O}

Keywords:

Rietveld method

Levenberg-Marquardt

Markov Chain Monte Carlo

Bayesian statistics

Dislocation densities

Stainless steels

\begin{abstract}
A B S T R A C T
We describe the analysis of in-situ HT-XRD data of a dual phase stainless steel exposed to a complex thermal cycle of heating, holding and cooling. For the conditions used only low quality diffraction data could be collected. Peak positions, peak areas and peak broadening are modeled by the Rietveld method. The low signal-to noise ratio and the presence of artificial peaks due to tube tails complicate the data evaluation. In a first attempt the parameters are refined by a local optimization procedure (e.g. Levenberg-Marquardt). However, this procedure fails by being caught in one of several local minima. Next, a Bayesian approach with a Markov Chain Monte Carlo (MCMC) algorithm is used as a global optimization procedure to refine the simulated Rietveld diffractograms. Accurate estimates of the evolution of the phase fractions and dislocation densities in martensite and austenite during all stages of the thermal cycle are obtained by this MCMC algorithm. While an approach based on multivariate second order Taylor series completely underestimates the error, the uncertainties in the model parameters could be estimated appropriately from histograms obtained by the MCMC method.
\end{abstract}

\section{Introduction}

The mechanical properties of polycrystalline materials strongly depend on their microstructure and in many cases this final microstructure is the outcome of several solid state phase transformations each generating their own transient microstructure. High-temperature $\mathrm{X}$-ray diffraction (HT-XRD) is a powerful technique to track these transient microstructures as a function of time and temperature. HT-XRD studies have been reported for different systems such as nickel-based [1,2], titanium-based [3], copper-based [4], iron-based [5,6], aluminium-based [7] and cobalt-based alloys [8], high-entropy alloys [9], metallic glasses [10] and finally ceramic and nano-ceramic materials [11]. All these diffraction data are characterized by peak positions (from which lattice parameters are derived), integral peak areas (from which phase fractions are derived) and peak broadening (from which crystallite size and dislocation densities are derived). All three parameters are not only a function of the actual microstructure at the time of measurement but also of instrumental components [12]. The microstructural changes such as the evolution of phase fractions and dislocation densities are commonly obtained using the Rietveld method [13-16] often in combination with the double Voigt peak broadening model. Alternative approaches for evaluating dislocation densities from X-ray diffraction data are based on Williamson-Hall equation or on the Warren-Averbach method (see e.g. [17,18]).

It is state of the art to optimize the Rietveld parameters for a certain diffractogram by a classical least-squares non-linear (local) minimizer. This approach is well-established and reliable results are obtained in a variety of different cases. However, the refinement of the Rietveld parameters may fail due to trapping in false minima as it is described in [19] and the likelihood of failure increases as the data quality decreases. That is why the Rietveld refinement procedure based on local optimization routines requires frequent interference by an expert during the refinement of each diffractogram (e.g. changing the initial parameter might be necessary). This complicates deriving microstructural changes obtained during processing of materials from in-situ measurements in case of limited sampling time per measurement and impedes automatic

\footnotetext{
* Corresponding author.

E-mail address: manfred.wiessner@anton-paar.com (M. Wiessner).
} 
evaluation of the diffractograms and finally, makes the ultimate results operator-expertise dependent.

It turns out that a reliable analysis of fit parameters of non-linear models is frequently only possible by means of global optimization tools. Global optimization is e.g. realized by means of adaptive Markov Chain Monte Carlo (MCMC) simulation algorithms [20-22], which are based on the Bayes theorem. It is demonstrated in [23-25] that this Bayesian approach can also be successfully applied to the Rietveld method. It is shown in this work that by applying the Bayes theorem on the Rietveld analysis not only lattice constants and phase fractions but also dislocation densities can be deduced from diffraction data even in case of a low signal-to-noise-ratio. The errors of these parameters can be estimated by using Probability Density Functions (PDF) obtained from the MCMC simulations.

\section{Theory}

There are two schools of thought on how best to analyse the complex information hidden in diffractograms and the subsequent Rietveld analysis; the classical or frequentist and the Bayesian statistics. Details about classical statistics can e.g. be found in [26], for the Bayesian approach the reader is referred to the textbook of Bolstad [27] and MacKay [28]. In the following both approaches are briefly discussed.

\subsection{Frequentist (classical) statistics}

The frequentist statistics are based on the idea that model parameters are fixed but unknown constants. Thus, probability statements cannot be made about their values. The statistical probability is based on the sampling distribution, e.g. by making more than one measurement under nominally identical conditions. In order to determine the best estimate of the mentioned model parameters the least squares approach is frequently used to quantitatively solve such regression problems [29]. It is nowadays standard to derive the confidence intervals of the model parameters and to calculate their covariance matrix [30] by means of commercially, publicly available or private software tools.

The starting point for the method of least squares is the objective function $S$, i.e. the sum of the weighted least squares (see Eq. (1)), which is to be minimized;

$S=\sum_{i=1}^{N} w_{i}\left(y_{i}-y_{c i}(\vec{\xi})\right)^{2}$

where $y_{i}$ is the $i^{\text {th }}$ of $n$ measuring points. The calculated quantity $y_{c i}(\vec{\xi})$ obtained from the model function and the weighting factor $w_{i}$ of each measuring point are further ingredients of the objective function $S$. The model parameters $\xi_{k}$ are summarized in the vector $\vec{\xi}$.

The weighted residuals $w_{i}\left(y_{i}-y_{c i}(\vec{\xi})\right)$ are normally distributed around 0 when

$w_{i}=\frac{1}{\sigma_{i}^{2}}$

where $\sigma_{i}$ is the standard deviation at point $i$.

The minimum $S$ is the optimal solution for the least-squares problem with respect to the model parameters $\xi_{k}$. The following partial differential equations vanish at the minimum [29]:

$\frac{\partial S}{\partial \xi_{k}}=0, k=1 \ldots p$ (number of parameters)

where $p<N$.

Inserting Eq. (1) in Eq. (3) yields

$-2 \sum_{i=1}^{n} w_{i}\left[y_{i}-y_{c i}(\vec{\xi})\right] \frac{\partial y_{c i}(\vec{\xi})}{\partial \xi_{k}}=0, k=1 \ldots p$ which is equal to

$\sum_{i=1}^{n} w_{i} y_{c i}(\vec{\xi}) \frac{\partial y_{c i}(\vec{\xi})}{\partial \xi_{k}}=\sum_{i=1}^{\mathrm{n}} w_{i} y_{i} \frac{\partial y_{c i}(\vec{\xi})}{\partial \xi_{k}}, k=1 \ldots p$

Eq. (4b) can be solved directly for linear problems, whereas nonlinear problems are solved iteratively. A guess vector $\vec{\xi}_{0}$ is initially introduced for the model parameters. The nonlinear equation $y_{\mathrm{ci}}(\vec{\xi})$ can be expressed by a Taylor series expansion around this guess vector by considering the linear terms only:

$y_{c i}(\vec{\xi})=y_{c i}\left(\overrightarrow{\xi_{0}}\right)+\sum_{l=1}^{p} \frac{\partial y_{c i}\left(\vec{\xi}_{0}\right)}{\partial \xi_{l}}\left(\xi_{l}-\xi_{0 l}\right)$

These terms (5) are inserted in Eq. 4b, yielding

$$
\begin{gathered}
\sum_{i=1}^{n} w_{i}\left(y_{\mathrm{c} i}\left(\vec{\xi}_{0}\right)+\sum_{l=1}^{p} \frac{\partial y_{\mathrm{ci} i}\left(\vec{\xi}_{0}\right)}{\partial \xi_{l}}\left(\xi_{l}-\xi_{0 l}\right)\right) \frac{\partial y_{\mathrm{c} i}(\vec{\xi})}{\partial \xi_{k}}= \\
=\sum_{i=1}^{n} w_{i} y_{i} \frac{\partial y_{\mathrm{c} i}\left(\vec{\xi}_{0}\right)}{\partial \xi_{k}}, k=1 \ldots p
\end{gathered}
$$

or, respectively

$$
\begin{aligned}
& \sum_{i=1}^{n} w_{i}\left(\sum_{l=1}^{p} \frac{\partial y_{c i}\left(\vec{\xi}_{0}\right)}{\partial \xi_{l}}\left(\xi_{l}-\xi_{0 l}\right)\right) \frac{\partial y_{c i}(\vec{\xi})}{\partial \xi_{k}}= \\
= & \sum_{i=1}^{n} w_{i}\left(y_{i}-y_{c i}\left(\vec{\xi}_{0}\right)\right) \frac{\partial y_{c i}\left(\vec{\xi}_{0}\right)}{\partial \xi_{k}}, k=1 \ldots p
\end{aligned}
$$

Eq. (6b) represents a linear system of equations that can be written in matrix form:

$\vec{A} \cdot\left(\vec{\xi}-\vec{\xi}_{0}\right)=\vec{\beta}$

$$
\text { with } \begin{aligned}
A_{l k} & =\sum_{i=1}^{n} w_{i} \frac{\partial y_{c i}\left(\vec{\xi}_{0}\right)}{\partial \xi_{l}} \cdot \frac{\partial y_{c i}\left(\vec{\xi}_{0}\right)}{\partial \xi_{k}}, \\
l & =1 \ldots p, k=1 \ldots p
\end{aligned}
$$

and $\beta_{l}=\sum_{i=1}^{n} w_{i}\left(y_{i}-y_{\mathrm{c} i}(\vec{\xi})\right) \frac{\partial y_{\mathrm{c} i}\left(\vec{\xi}_{0}\right)}{\partial \xi_{l}}, l=1 \ldots p$

As mentioned above, the model parameters are obtained iteratively, and are summarized in a vector $\vec{\xi}$. It is not guaranteed that the global minimum of the objective function $S$ is reached in case of non-linear problems, i.e. the linearization of the problem might be inappropriate. Different initial guess vectors $\vec{\xi}_{0}$ can result in finding different local minima for non-linear problems. Local convergence is not guaranteed when a simple Gauss-Newton approach is used. In recent times more robust methods e.g. the Levenberg-Marquardt [31,32] algorithm, are frequently exploited, where the Gauss-Newton approach is combined with the method of gradient descent, but also this method does not always lead to the true minimum and sometimes local minima rather than the global minimum are found. Hence, the global Bayesian approach has become increasingly popular as an alternative method for solving optimization problems.

\subsection{Bayesian statistics}

In contrast to the frequentist approach, the Bayesian approach is based on the assumption that the parameter values are uncertain and they are treated as random variables. Therefore, rules of probability can 
be applied directly. Any prior knowledge of the parameter set (e.g. phase fractions and dislocation densities must be positive values by default) is considered in setting the prior probability distribution $P(\vec{\xi} \mid H)$ for the complete hypothesis space $H$. New data $D$ are considered in the likelihood function $P(D \mid \vec{\xi}, H)$ that changes the prior distribution. The likelihood function $P(D \mid \vec{\xi}, H)$ is the conditional probability for a parameter set of the model to describe the experimental data. As a result the posterior probability distribution $P(\vec{\xi} \mid D, H)$ of the model parameters follows from the prior distribution by considering the Likelihood function $P(D \mid \vec{\xi}, H)$ via the Bayesian theorem [27,28,33]:

$P(\vec{\xi} \mid D, H)=\frac{P(D \mid \vec{\xi}, H) \cdot P(\vec{\xi} \mid H)}{P(D \mid H)}$

The conditional probability $P(D \mid \vec{\xi}, H) \cdot P(\vec{\xi} \mid H)$ is normalized by the evidence $P(D \mid H)$ according to Eq. (8). In this work a "flat" prior is introduced, i.e. the same probability is assumed for all possible values of the parameters. For this case, the posterior probability and likelihood are proportional. According to [28] Eq. (8) can be simplified for normalized probabilities to:

$P(\vec{\xi} \mid D, H)=P(D \mid \vec{\xi}, H)$

The hypothesis space $H$ is constant (i.e. a certain model is used throughout the whole calculation). Thus, the distribution $P(D \mid H)$ (evidence) remains also constant [33].

The posterior distribution of the model parameters (Eq. 9) can be calculated numerically by e.g. using the Metropolis-Hastings algorithm [34,35]. This Metropolis-Hastings algorithm is a subclass of Markov Chain Monte Carlo (MCMC) methods, which is widely used for high-dimensional problems [28]. Despite the fact that an element of the generated sequence depends on the previous element, the Markov chain generates a representative random sample from the probability distribution. In a first step a new parameter state is determined from the previous distribution located close to the old state. In a second step, the ratio of the probabilities of a possible new state and the old state is determined. The new parameter set is always accepted for a ratio greater than 1 , meaning that the new state has a higher probability than the old one. For a ratio smaller than 1 , the possible new state is accepted with a probability given by that ratio. Based on this ratio, the decision is made by an equally distributed random generator. In case that the new state is rejected, the old state is inherited. Based on this method it is possible to reach regions in the parameter space which are rather unlikely, and thereby it is also possible to escape from local minima. Histograms are plotted from the values of each parameter saved in the Markov chain. The frequencies in the value ranges of the parameters correspond to the probability distribution of these model parameters (ergodicity of Metropolis-Hastings algorithms). In this work the effective and robust DREAM (D) algorithm, based on an optimized MCMC method, is employed for the numerical analysis [20-22].

\subsection{Comparison of classical and Bayesian statistics}

It is one of the goals of this paper to find the probability distribution $P(\vec{\xi} \mid \vec{y}, H(\vec{\sigma}))$ of the model parameters, where the vector $\vec{y}$ corresponds to the new dataset $D$. The hypothesis space $H$ contains the vector $\vec{\sigma}$ of the standard deviations of all measuring points. On the one hand, according to the formula Eq. 9, this probability distribution $P(\vec{\xi} \mid \vec{y}, H(\vec{\sigma}))$ equals the probability $P(\vec{y} \mid \vec{\xi}, H(\vec{\sigma}))$ for a flat prior distribution; $P(\vec{\xi} \mid \vec{y}, H(\vec{\sigma}))=P(\vec{y} \mid \vec{\xi}, H(\vec{\sigma}))$. On the other hand, the probability $P(\vec{y} \mid \vec{\xi}, H(\vec{\sigma}))$ represents the cumulative probability of the
Table 1

Chemical composition of the martensitic stainless steel.

\begin{tabular}{llllllll}
\hline Component & $\mathrm{C}$ & $\mathrm{Si}$ & $\mathrm{Mn}$ & $\mathrm{Cr}$ & $\mathrm{Mo}$ & $\mathrm{Ni}$ & $\mathrm{N}$ \\
\hline Mass fraction in $\%$ & 0.04 & 0.40 & 0.40 & 15.4 & 0.90 & 5.30 & 0.04 \\
\hline
\end{tabular}

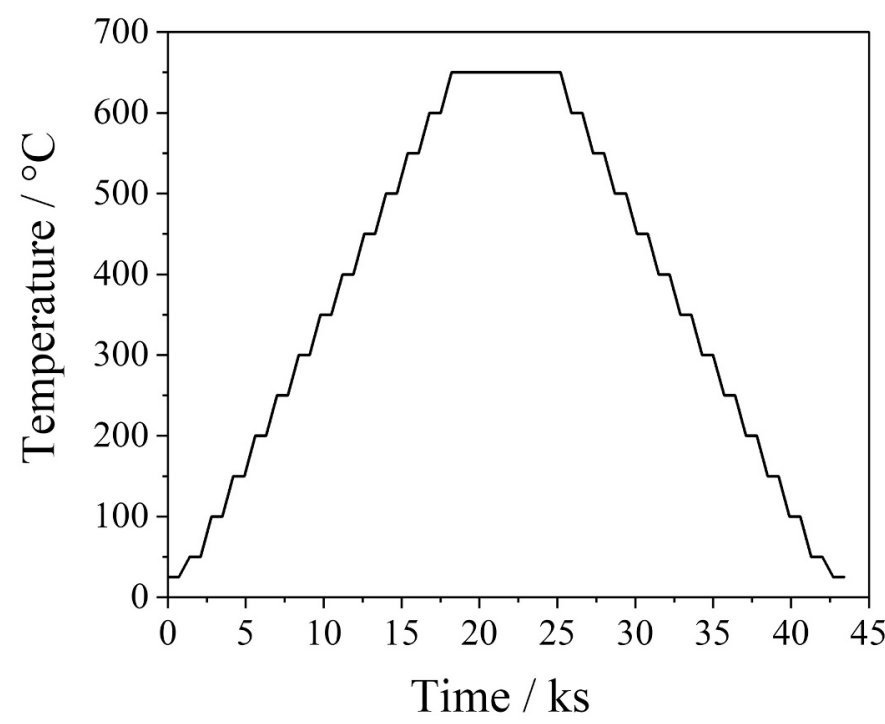

Fig. 1. Time-Temperature curve for the in-situ heat treatment during the XRD measurements.

individual measuring points achieved by multiplying the probability of the parameters of each data point in the Bayesian framework [28,33]:

$P(\vec{\xi} \mid \vec{y}, \vec{\sigma})=\prod_{i=1}^{n} \frac{1}{\sqrt{2 \pi} \sigma_{i}} \exp \left(-\frac{\left(y_{i}-y_{\mathrm{c} i}(\vec{\xi})\right)^{2}}{2 \sigma_{i}^{2}}\right)$

In the framework of (Eq. 10) the correlation between neighboring points is assumed to be negligible. In addition, the experimental values are approximated by a Gaussian distribution, since for a sufficiently high number $n$ of measuring points $(n>10)$ the Poisson distribution converges to a Gaussian distribution. Thus, the standard deviations $\sigma_{i}$ of each experimental point $y_{i}$ are approximated by the square roots of these values $y_{i}$ [19]. In [23,24], for reasons of simplicity, the deviation between measurement and simulation of each point is approximated by a fixed average standard deviation (same weighting factor) for all measurement points. A drawback of this approach is that pronounced peaks are overestimated.

The Bayesian probability density distribution $P(\vec{\xi} \mid \vec{y}, \vec{\sigma})$ (Eq. 10) can be compared to the objective function $S$ (Eq. 1) of the least squares method;

$\ln (P(\vec{\xi} \mid \vec{y}, \vec{\sigma}))=-\frac{n}{2} \ln (2 \pi)-\sum_{i=1}^{n} \ln \sigma_{i}-\frac{1}{2} S$

$S=\sum_{i=1}^{n} \frac{\left(y_{i}-y_{c i}(\vec{\xi})\right)^{2}}{\sigma_{i}^{2}}$

in case that the reciprocal variances in the probability distribution are set equal to the weighting factors in the classical least squares approximation. Thus, the minima found by the least squares method applied to $S$ correspond to the maxima in the probability distribution.

\section{Experiments and their evaluation by Rietveld method}

In-situ HT-XRD experiments are performed by means of a D8 

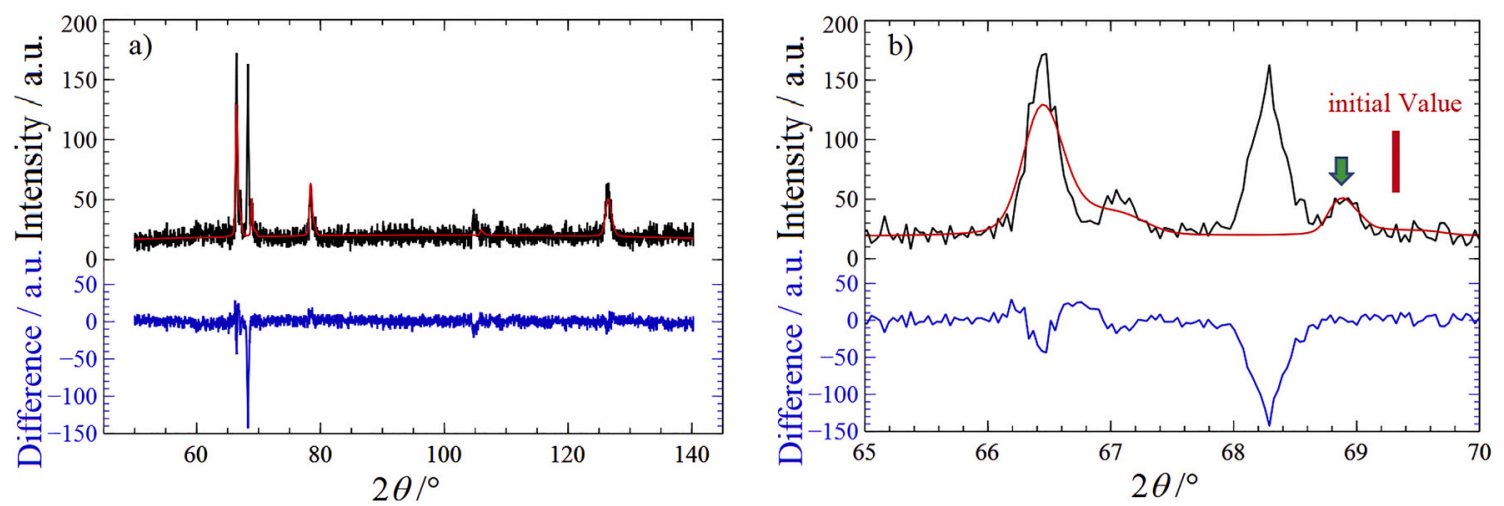

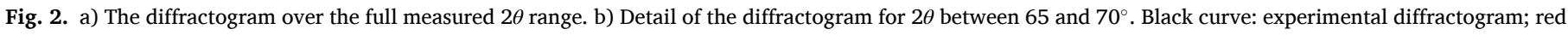

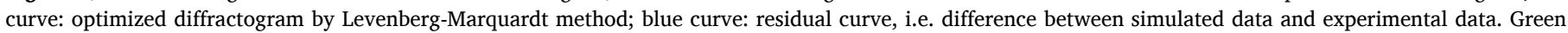

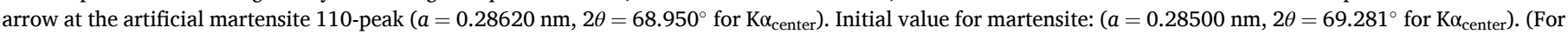
interpretation of the references to colour in this figure legend, the reader is referred to the web version of this article.)

Advance diffractometer (Bruker, Germany) in parallel beam diffraction geometry using $\mathrm{Cr}-\mathrm{K} \alpha$ radiation $\left(\mathrm{Cr}-\mathrm{K} \alpha_{1}=0.228975 \mathrm{~nm}, \mathrm{Cr}-\mathrm{K} \alpha_{2}=\right.$ $\left.0.229366 \mathrm{~nm}, \mathrm{Cr}-\mathrm{K} \alpha_{\text {center }}=0.229105 \mathrm{~nm}\right)$ and an attached hightemperature chamber HTK 1200 (Anton Paar GmbH, Austria). A martensitic stainless steel sample (Böhler steel grade N404 [36]) is investigated. The chemical composition of this steel is provided in Table 1 . The dimensions of the cylindrical samples are specified by a height $h=1.2 \mathrm{~mm}$ and a diameter $d=14 \mathrm{~mm}$.

First, the steel sample is heated with a rate of $0.82 \mathrm{Ks}^{-1}$ and then fully austenitized at $1010^{\circ} \mathrm{C}$ inside the high temperature chamber HTK 1200 in an inert gas atmosphere $\left(\mathrm{N}_{2}\right)$ for a dwell time of $600 \mathrm{~s}$. Then, the sample is freely cooled with a cooling time $t_{8 / 5}=300 \mathrm{~s}$ to room temperature. After this initial heat treatment the sample is polished in order to remove a possible thin oxide layer with a diamond paste of $9 \mu \mathrm{m}, 3 \mu \mathrm{m}$ and then $1 \mu \mathrm{m}$. Finally, the sample is treated with an amorphous $0.02 \mu \mathrm{m}$ colloidal silica suspension using the VibroMet apparatus from Buehler, Germany.

Then, the sample is subjected to the tempering treatment shown in Fig. 1. The sample is heated from room temperature to $650^{\circ} \mathrm{C}$ in steps of $50 \mathrm{~K}$ with an effective heating rate of $3.57 \cdot 10^{-2} \mathrm{~K} \mathrm{~s}^{-1}$ up to a tempering temperature of $650{ }^{\circ} \mathrm{C}$, then kept there for $7 \cdot 10^{3} \mathrm{~s}$ and subsequently cooled down with the cooling rate of $3.57 \cdot 10^{-2} \mathrm{~K} \mathrm{~s}^{-1}$ steps of $50 \mathrm{~K}$ until room temperature is reached again. The time for recording one diffractogram is $700 \mathrm{~s}$. The diffractograms are recorded continuously, i.e. during each heating step of $50 \mathrm{~K}$ one diffractogram is recorded, and another diffractogram is recorded during each intermediate holding step. Ten further diffractograms are recorded during tempering at
$650{ }^{\circ} \mathrm{C}$. For the cooling the same procedure is applied as during heating. The Rietveld method using the fundamental parameter model in combination with the Double-Voigt approach is used to simulate diffractograms for specific model parameters, e.g. lattice parameters, phase fractions and dislocation densities [12-16]. The fitted lattice parameters correspond to the $2 \theta$ - angles via the Bragg equation by means of the Rietveld method. The phase fractions are obtained by evaluating the integral intensities of the simulated peaks, while taking into account the Lorentz-Polarisation factor, the structure factor and the multiplicity from multiple equivalent planes. The dislocation densities in both martensite and austenite are determined by the same approach as used in [16]. It is demonstrated in [37] that the integral breadth $\beta_{\text {strain }}$ in the $2 \theta$ - space and the microstrain $e$ are related by Eq. (12):

$\beta_{\text {strain }}=4 \operatorname{etan} \theta$ with $e=\frac{\Delta d}{d}$

In this work it is tacitly assumed that stresses and strains follow an isotropic material behavior, i.e. the anisotropy of the crystal lattice is neglected.

As described in [38] the dislocation density $\rho_{i}$ is proportional to the square of the microstrain $e_{i}$ :

$\rho_{i}=\rho_{0, i} e_{i}^{2}$

The evaluation of the proportionality factor $\rho_{0, i}$ of the dislocation density $\rho_{i}$ of phase $i$ in cubic systems is derived in [16]:
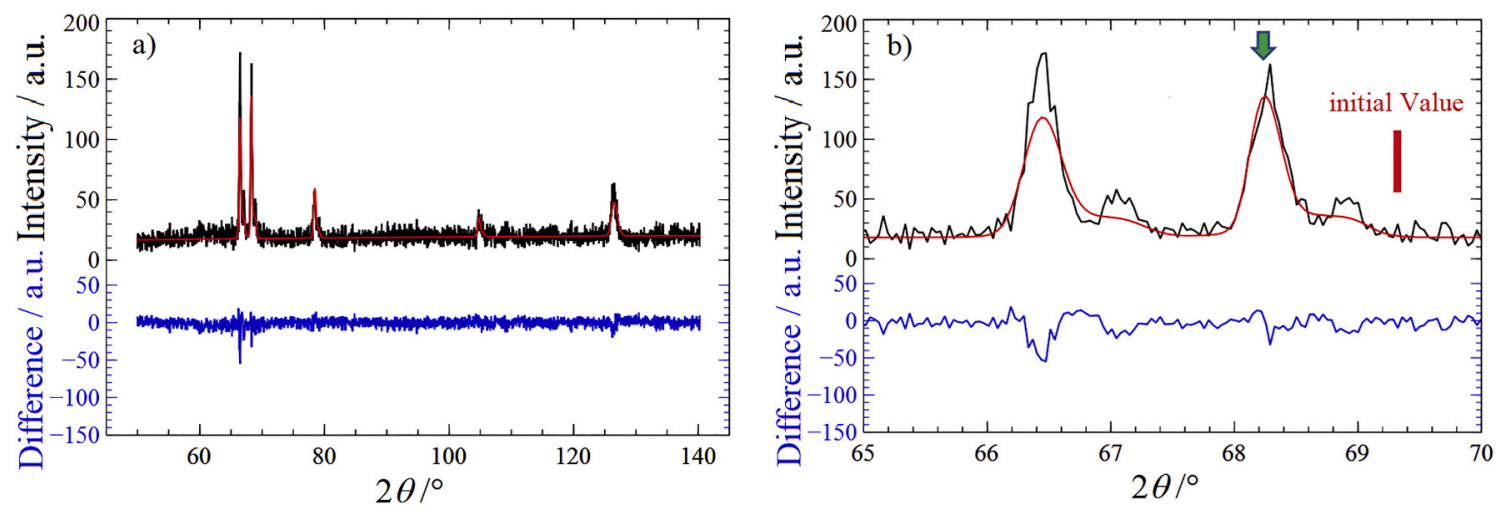

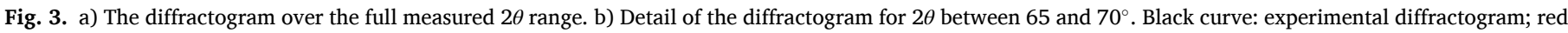

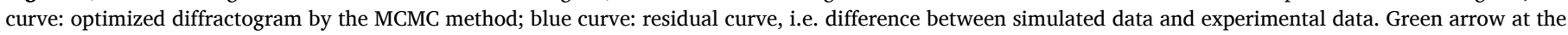

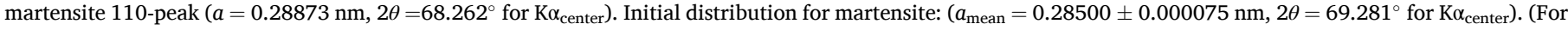
interpretation of the references to colour in this figure legend, the reader is referred to the web version of this article.) 
$\rho_{0, i}=\frac{6\left(1+v_{i}\right)}{\left(C_{i} a_{i}\right)^{2} \cdot\left(1+2 v_{i}^{2}\right)}$

$$
\text { with }
$$

$C_{1}=\frac{\sqrt{3}}{2}$ for the bcc lattice

$C_{2}=\frac{\sqrt{2}}{2}$ for the fcc lattice

based on the assumption that $\rho_{0, i}$ is a function of the lattice constant $a_{i}$ and Poisson's ratio $\nu_{i}$, only. The lattice constant $a_{i}$ and the microstrain $e_{i}$ are obtained from evaluating the diffractograms, and the temperaturedependent Poisson's ratio $\nu_{i}$ can be found in [39].

Instrumental effects are considered by applying the fundamental parameter model [12] (which is frequently used as a part of the total Rietveld method) when analyzing the experimental raw data. The parameters of this model are determined from diffraction measurements on nearly defect-free cubic $\mathrm{LaB}_{6}$ powder (standard: NIST 660a [40]).

Two methods are used to optimize the simulated Rietveld diffractograms: The Levenberg-Marquardt [31,32] approach which is a (single shot) local optimization procedure and the global Bayesian approach with a Markov Chain Monte Carlo (MCMC) algorithm. Hundreds of linked Markov chains are created within the DREAM (D) algorithm used. The first hundred cycles are rejected (burn-in period) from each chain. The next 500 cycles from each chain are used for parameter extraction. The resulting model parameters and their uncertainties from the two models are compared.

\section{Results and discussion}

\subsection{Refinement by Levenberg-Marquardt and Monte Carlo differential evolution simulation}

We like to start by showing the experimental diffractograms and the simulated diffractograms obtained from the optimized model parameters both optimization techniques, LM (see Fig. 2) and MCMC (see Fig. 3) for one particular state during a complex thermal protocol leading to many completely different transient microstructures. Experimental data correspond to a diffractogram recorded at the end of the isothermal heat treatment (temperature $\vartheta=650{ }^{\circ} \mathrm{C}$, time $t=25.2 \cdot 10^{3} \mathrm{~s}$ ). This condition is selected because at this tempering temperature various microstructural changes occur simultaneously. Martensite transforms to a high volume fraction of reverted austenite combined with a dramatic decrease of the dislocation density in martensite. While being complex structure to analyse anyway, the tube tail effect complicates the optimization analysis for finding the global minimum during the whole heat treatment (i.e. also for high volume fractions of martensite), due to an objective function containing many local minima.

Diffractograms with low signal to noise levels were obtained as a result of the short measurement times and the measurement configuration used (see Fig. 2a). The average value of these signal to noise levels is approximated by $\sqrt{N}=4.6$ for an average value of $N=21$ counts per point (see Fig. 2a). The peak height measured of only 172 counts per condition is well below the height recorded during conventional XRD experiments under more gently varying conditions where $10^{4}$ to $10^{5}$ counts per point are standard. It is known that counting statistics errors [41] are not negligible compared to unknown systematic model errors $[42,43]$ for powder diffractograms recorded with intensities of only a few thousand counts. This low count rate makes data analysis a complex and non-trivial problem.

Let us now look in more detail at the differences obtained when applying both optimization methods: When using the LevenbergMarquardt algorithm with an initial starting value for the martensite lattice parameter of $a=0.28500 \mathrm{~nm}\left(2 \theta=69.281^{\circ}\right.$ for $\mathrm{K} \alpha_{\text {center, }}, 2 \theta=$ $69.236^{\circ}$ for $\mathrm{K} \alpha_{1}$, for $\mathrm{hkl}=110$ and $\mathrm{Cr} \mathrm{K} \alpha_{1}$ ) the martensite peaks in the optimized simulated diffractogram (red line in Fig. 2a) are trapped in the artificial martensite peak caused by the tube tail marked by the green arrow. Due to the tube tail effect the real martensite peak for

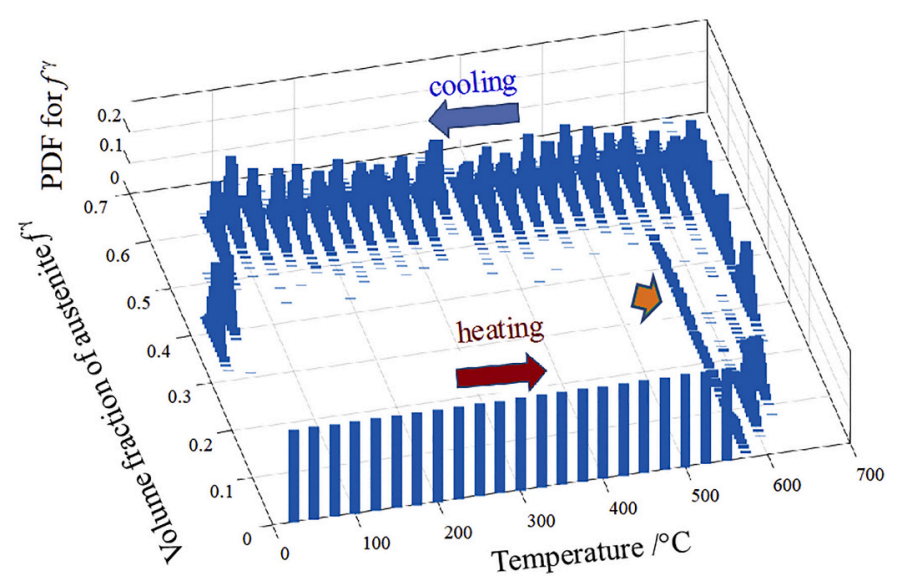

Fig. 4. PDF for the austenite volume fraction $f^{\prime}$ during the whole tempering process as a function of the volume fraction of austenite (orange arrow: first detected formation of reverted austenite) at different temperatures during heating, isothermal holding and cooling. (For interpretation of the references to color in this figure legend, the reader is referred to the web version of this article.)

$\mathrm{K} \alpha_{\text {center }}$ at $2 \theta=68.262^{\circ},\left(2 \theta=68.217^{\circ}\right.$ for $\left.\mathrm{K} \alpha_{1}\right)$ remains undetected. It is worth mentioning that $\mathrm{K} \alpha_{\text {center }}$ had to be introduced as it is impossible to separate the $\mathrm{K} \alpha_{1}, \mathrm{K \alpha}_{2}$ martensite peaks at the $2 \theta$ angles investigated. The value of $K \alpha_{\text {center }}$ can be directly read from the magnified section shown in Fig. 2b.

Now let us turn to the MCMC analysis. The initial lattice parameter distribution of martensite of each Markov chain is characterized by its mean value $\left(a_{\text {mean }}=0.28500 \mathrm{~nm}\right)$, which equals the value used in the $\mathrm{LM}$ algorithm but allowing a standard deviation $\left(\sigma=7.5 \cdot 10^{-5} \mathrm{~nm}\right)$. The martensite lattice parameter of the Markov chains evolves to the correct martensite peak for the majority of chains within less than one hundred cycles. The most probable MCMC-based simulated diffractogram is presented in Fig. 3 (red line). A martensite lattice parameter distribution $a=(0.28873 \pm 0.00007) \mathrm{nm}$, correponding to $\mathrm{Cr} \mathrm{K} \alpha_{\text {center }}$ at $2 \theta=$ $68.262^{\circ},\left(\mathrm{K} \alpha_{1} \text {-peak at } 68.217 \pm 0.020\right)^{\circ}$ for $\left.\mathrm{hkl}=110\right)$, is obtained by this MCMC-approach (green line in Fig. $3 \mathrm{~b}$ ) and is considered to be the true global minimum. The residuals, i.e. the differences between the simulated diffractograms and the experimental intensities as a function of $2 \theta$ are depicted as blue lines in Fig. 3a and b. The residuals for the MCMC-method shown in Fig. 3 are acceptable over the full $2 \theta$-range, whereas the residuals presented in Fig. 2 (LM approach) strongly deviate for the martensite peaks (martensite lattice parameter $\quad a=0.28620$ $\mathrm{nm}$ by Levenberg-Marquardt approach versus martensite lattice parameter $a=0.28873 \mathrm{~nm}$ obtained by MCMC approach).

\subsection{Determination of phase fractions}

Let us monitor the evolution of the MCMC obtained posterior probability density function (PDF) of the austenite volume fraction during the whole heat treatment process as presented in Fig. 4. At the beginning of the in-situ experiment, i.e. during heating, the volume fraction $f^{\prime}$ of austenite is below the detection limit $\left(f^{\prime} \leq 0.05\right.$ for these in-situ experiments). The probability density is zero everywhere, but mathematically goes to infinity at a phase fraction of austenite being zero. As a consequence the posterior PDF of the austenite volume fraction is classified as a Dirac delta function in the range of very small austenite phase fractions which continues from the starting temperature of $25^{\circ} \mathrm{C}$ to a temperature of $575{ }^{\circ} \mathrm{C}$ (Fig. 4) where the first signs of austenite formation are recorded.

Detectable fractions of reverted austenite occur during heating above a temperature of $575{ }^{\circ} \mathrm{C}$. A wide probability distribution is obtained for the austenite phase fraction directly at $575{ }^{\circ} \mathrm{C}$ (see orange arrow in Fig. 4), i.e. the value for the volume fraction of austenite is highly 


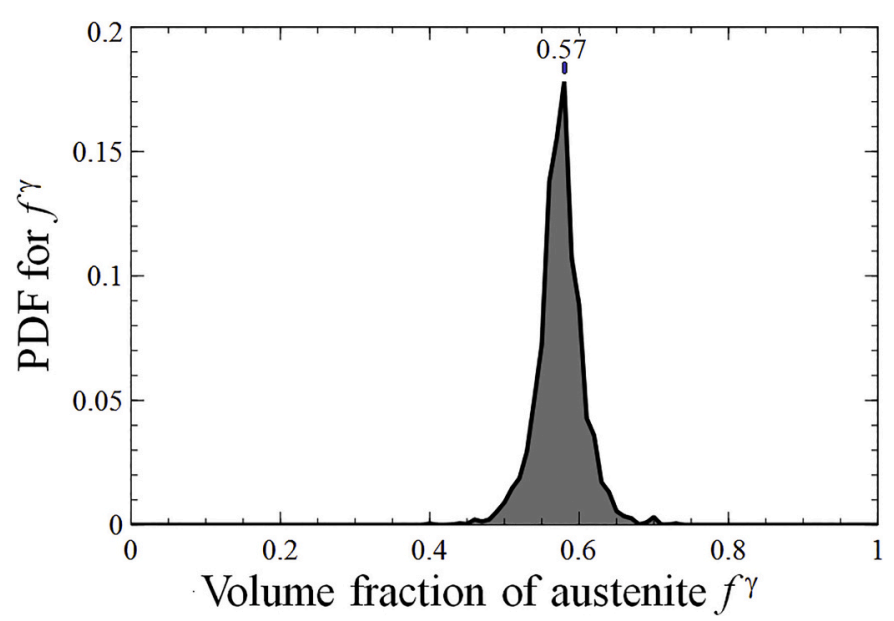

Fig. 5. PDF for $f^{\prime}$ at the end of the isothermal step at $650{ }^{\circ} \mathrm{C}$ (same condition as used in Figs. 2 and 3).

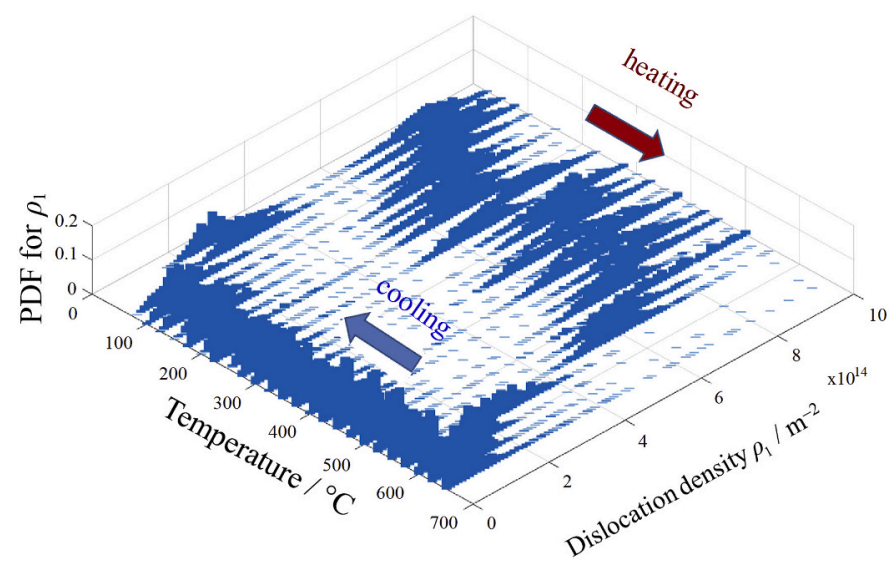

Fig. 6. PDF of the dislocation density $\rho_{1}$ of martensite during the whole tempering process.

uncertain at this stage of the heat treatment. The PDF is shifted to higher values of the volume fraction of reverted austenite for increasing temperatures higher than $575{ }^{\circ} \mathrm{C}$. The full-width-half-maximum of the PDF becomes smaller. The maximum volume fraction of reverted austenite further increases to $0.57 \pm 0.05$ (error is given by one standard deviation) during isothermal holding at $650{ }^{\circ} \mathrm{C}$ at the end of the isothermal step (i.e. the condition also presented in Figs. 2 and 3. The austenite fraction probability distribution at this stage of the heat treatment is shown in detail in Fig. 5. The volume fraction of austenite remains constant during cooling process until $100{ }^{\circ} \mathrm{C}$ is reached (Fig. 4). A partial retransformation to martensite occurs during cooling below $100{ }^{\circ} \mathrm{C}$ resulting in a final austenite volume phase fraction of approximately 0.40 .

As a result of the solution according to the Levenberg-Marquardt optimization procedure being trapped in a local minimum, the calculated phase fractions strongly deviate from their "true" values. For the diffractogram presented in Fig. 2 (i.e. at the end of the isothermal annealing step at $650{ }^{\circ} \mathrm{C}$ ) the Levenberg-Marquardt approach suggests an value for the volume fraction $f^{\alpha^{\prime}}$ of martensite, $f^{\alpha^{\prime}}=0.15$ and of austenite $f^{\prime}=1-f^{\alpha^{\prime}}=0.85$. The value $f^{\alpha^{\prime}}=0.15$ for the martensite fraction is underestimated by the Levenberg-Marquardt approach. The value obtained by the MCMC method is $f^{\alpha^{\prime}}=0.43$ and of austenite $f^{\prime}=$ $1-f^{\alpha^{\prime}}=0.57$ (see Figs. 4 and 5).

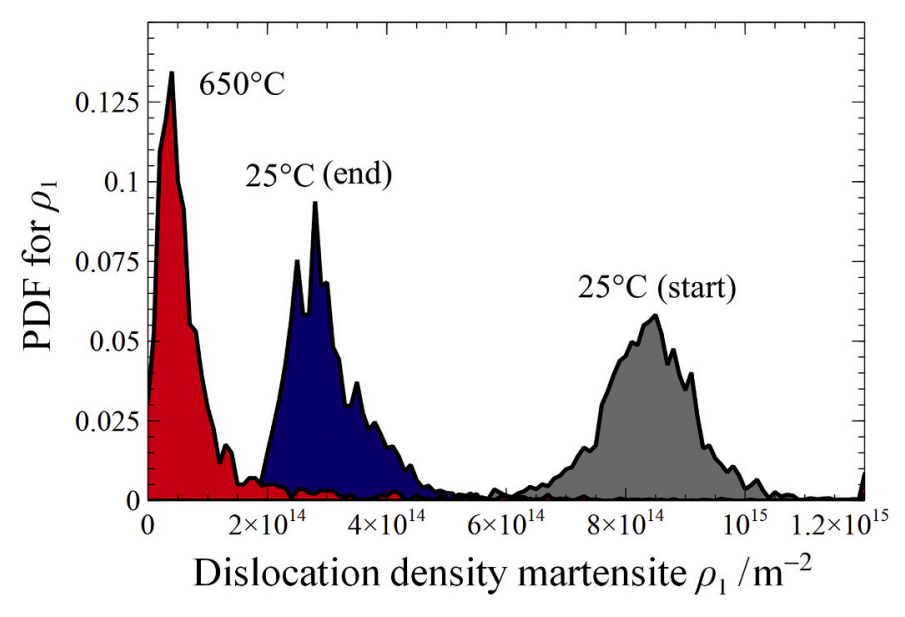

Fig. 7. PDF of the dislocation density $\rho_{1}$ of martensite at the start of the heat treatment at $25{ }^{\circ} \mathrm{C}$ (gray), at the end of the isothermal step at $650{ }^{\circ} \mathrm{C}$ (red) and at the end of thermal cycle at $25{ }^{\circ} \mathrm{C}$ (darkblue). (For interpretation of the references to colour in this figure legend, the reader is referred to the web version of this article.)

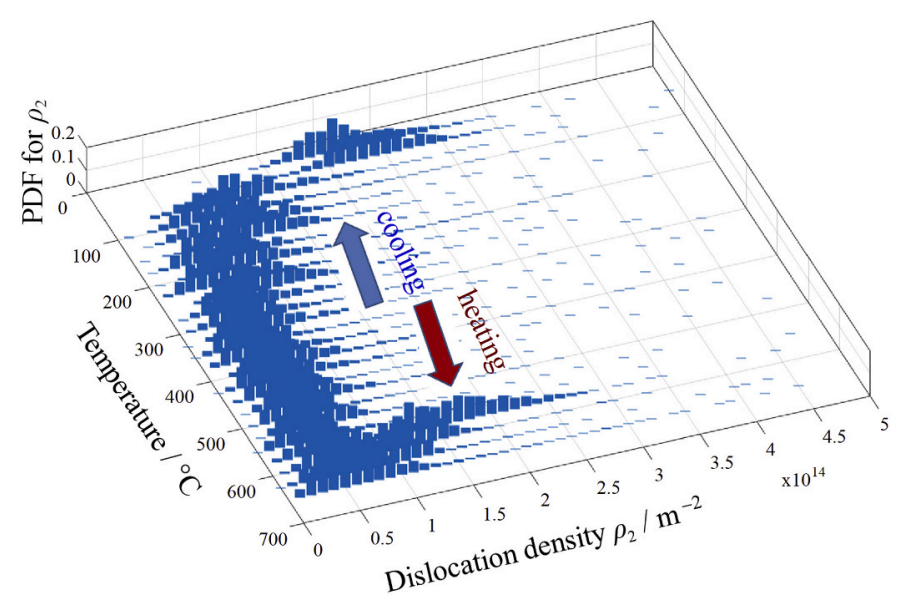

Fig. 8. PDF of the austenite dislocation density $\rho_{2}$ during the whole tempering process.

\subsection{Determination of dislocation densities in martensite and austenite}

As described in the previous section the LM-method with initial parameters that do not converge to the global minimum, results in a wrong phase fraction and will lead to unrealistic dislocation densities in martensite and austenite. In the following, the low signal-to-noise ratio diffractograms are analyzed by the Bayesian based Markov Chain Monte Carlo (MCMC) algorithm. The PDF of the dislocation density of martensite is evaluated for the whole tempering process and plotted versus temperature in Fig. 6.

During heating at temperatures below $550{ }^{\circ} \mathrm{C}$ the maximum value of the PDF of the dislocation density $\rho_{1}$ of martensite is comparatively high (approximately $8 \cdot 10^{14} \mathrm{~m}^{-2}$ ). During further heating and holding at $650{ }^{\circ} \mathrm{C}$ annealing effects occur which reduce the lattice defect density in martensite due to annihilation of dislocations. The maximum value of the PDF of the dislocation density $\rho_{1}$ of martensite remains at a low value of approximately $0.4 \cdot 10^{14} \mathrm{~m}^{-2}$ during cooling, but increases below $100{ }^{\circ} \mathrm{C}$ during the partial retransformation of austenite to martensite.

In Fig. 7 , the PDF of the dislocation density of martensite $\rho_{1}$ is plotted at selected special time periods, at the beginning of the heat treatment at $25^{\circ} \mathrm{C}$ (gray area), at the end of the isothermal step at $650{ }^{\circ} \mathrm{C}$ (red area) and after heat treatment at $25{ }^{\circ} \mathrm{C}$ (blue area). The maximum of the PDF 


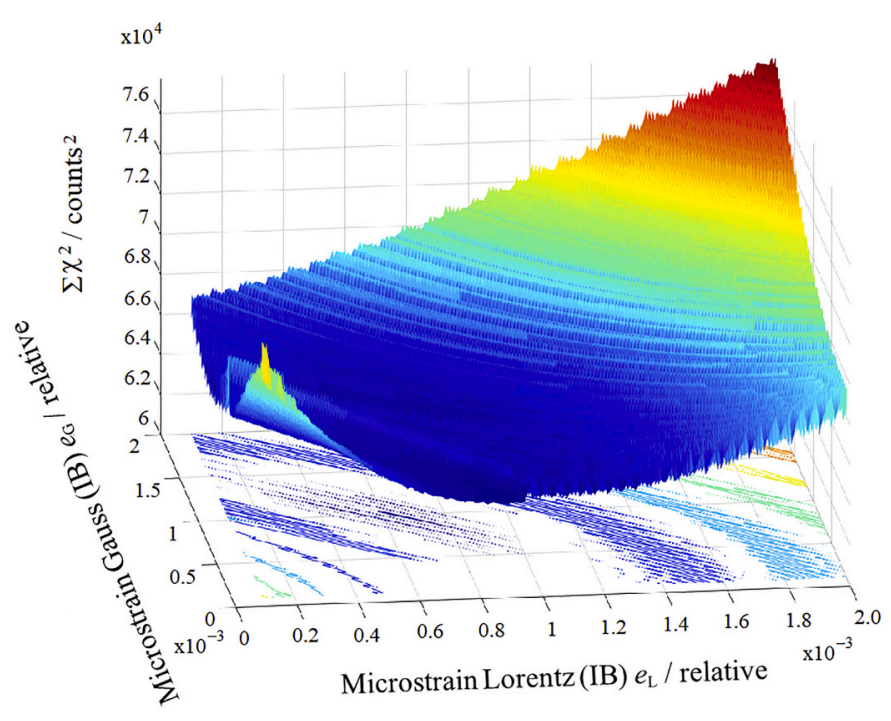

Fig. 9. The sum of the least square residuals $\Sigma \chi^{2}$ as a function of the microstrains $e_{\mathrm{L}}$ and $e_{\mathrm{G}}$ of martensite at the end of the isothermal step at $650{ }^{\circ} \mathrm{C}$.

of the dislocation density of martensite $\rho_{1}$ is $8.5 \cdot 10^{14} \mathrm{~m}^{-2}$ at $25{ }^{\circ} \mathrm{C}$ (start), $0.4 \cdot 10^{14} \mathrm{~m}^{-2}$ at the end of the isothermal step at $650{ }^{\circ} \mathrm{C}$ and $2.8 \cdot 10^{14} \mathrm{~m}^{-2}$ after heat treatment at $25^{\circ} \mathrm{C}$.

In addition the evolution of the dislocation density of austenite during this thermal treatment is presented. As demonstrated in Fig. 4, the austenite fraction remains below the detection limit at temperatures below $550{ }^{\circ} \mathrm{C}$ during the heating procedure. The integral breadth of the austenite peaks is determined with a high uncertainty during heating at $575{ }^{\circ} \mathrm{C}$ and $600{ }^{\circ} \mathrm{C}$, which goes along with the broad PDFs of the austenite dislocation density $\rho_{2}$ (see Fig. 8). A strong increase in the dislocation density $\rho_{2}$ is observed below $100{ }^{\circ} \mathrm{C}$ during cooling due to retransformation of austenite to martensite. This increase of the dislocation density $\rho_{2}$ during cooling is a consequence of transformation strains that occur due to the volume mismatch of austenite and martensite. While metallurgically this generation of new dislocations in both the martensite and the austenite, is well established, the fact that it could be determined under the present conditions is a major step forward in the XRD field.

\subsection{Estimation of the error of the dislocation densities}

The dislocation densities $\rho_{1}$ and $\rho_{2}$ in martensite and austenite are calculated from the experimentally observed peak broadening angles (integral breadth) $\beta_{\text {strain,1 }}$ and $\beta_{\text {strain, } 2}$ for the martensite and austenite peaks via inverse modelling by introducing the microstrains $e_{1}$ and $e_{2}$ (see Eq. 12). Each of the microstrains $e_{1}$ and $e_{2}$ are calculated as the convolution of a Gauss-function (microstrain $e_{\mathrm{G}}$ ) and a Lorentz-function (microstrain $e_{\mathrm{L}}$ ), where $e_{\mathrm{G}}$ and $e_{\mathrm{L}}$ are the values of the integral breadth for the normalized Gauss function and the normalized Lorentz-function, respectively. A good approximation of the microstrain $e$ is a pseudoVoigt function $[14,44,45]$ which is a sum of appropriate Lorentz and Gauss components.

$e=f\left(\mathrm{e}_{\mathrm{L}}, e_{\mathrm{G}}\right)$

It is demonstrated in the following that the relative estimated error (uncertainty) of the microstrain $\frac{\sigma_{e}}{e}$ and the resulting relative error of the dislocation densities $\frac{\sigma_{\rho}}{\rho}$ e.g. for martensite are dramatically underestimated when using classical error propagation. To this end the sum of the least square residuals $\Sigma \chi^{2}$ is plotted as a function of the microstrains $e_{\mathrm{L}}$ and $e_{\mathrm{G}}$ of martensite at the end of the isothermal step at $650{ }^{\circ} \mathrm{C}$ (Fig. 9).

A multitude of local minima occur close to each other by varying the

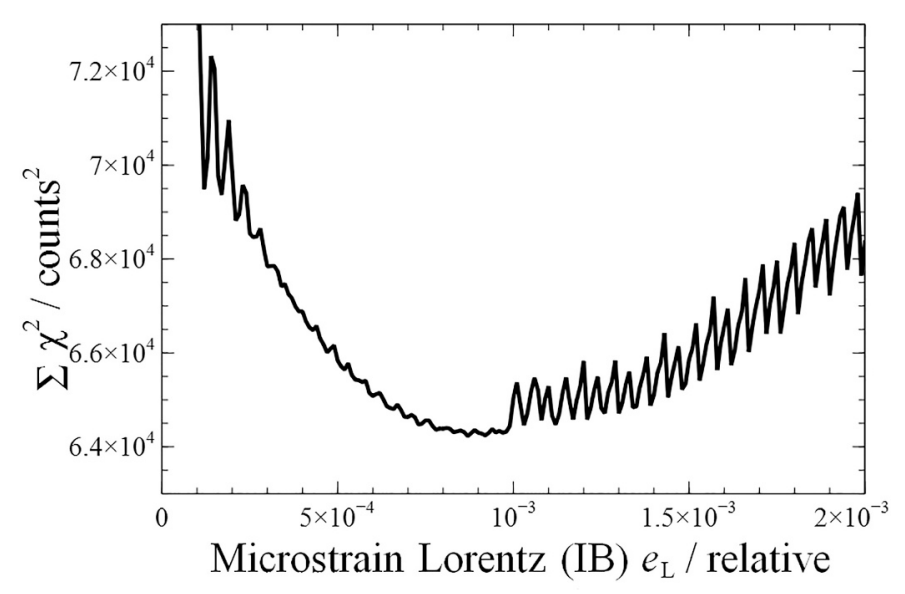

Fig. 10. $\Sigma \chi^{2}$ versus microstrain $e_{\mathrm{L}}$ at the end of the isothermal step at $650{ }^{\circ} \mathrm{C}$ in martensite.

values of the Lorentz and Gauss components of the microstrain $e$. The presence of many local minima is shown more clearly in a vertical section of the plot in Fig. 9 at a constant microstrain $e_{\mathrm{G}}=0.0393 \cdot 10^{-3}$, i.e. $\Sigma \chi^{2}$ versus microstrain $e_{\mathrm{L}}$ is presented in Fig. 10 .

It is clear that fluctuations occur in the $\Sigma \chi^{2}\left(e_{\mathrm{L}}\right)$-function. For classical error estimation, $\Sigma \chi^{2}$ is approximated by a $2^{\text {nd }}$ order Taylor series at the global minimum of the $n$ fit parameters $\overrightarrow{\xi_{0}}$. The partial first derivatives of $\Sigma \chi^{2}$ with respect to the fit parameters become zero. The $2^{\text {nd }}$ order Taylor series are of the following form,

$\sum \chi^{2}(\vec{\xi})=\sum \chi^{2}\left(\overrightarrow{\xi_{0}}\right)+\frac{1}{2}\left(\vec{\xi}-\overrightarrow{\xi_{0}}\right)^{T} \vec{H}\left(\vec{\xi}-\overrightarrow{\xi_{0}}\right)$

with $H_{i j}=\frac{\sum \chi^{2}\left(\overrightarrow{\xi_{0}}\right)}{\partial \xi_{i} \partial \xi_{j}}$ being the Hessematrix. Details concerning Eq. (14) can be found in [31].

According to Figs. 9 and 10 the range in which the Taylor series is applicable is severely limited due to strong fluctuations of $\Sigma \chi^{2}$. Thus, the classical error estimation is not applicable for this analysis. It is demonstrated in the following that in case of the error of the microstrain $e$ and the dislocation density $\rho$ of martensite and austenite that the calculated values are unrealistic. The Hessematrix $H_{i j}$ is calculated numerically via the corresponding second derivatives of $\Sigma \chi^{2}$. By such an approach according to Eq. (10), the probability $P \propto \exp \left(-\Sigma \chi^{2}\right)$ with $p$ fit parameters is approximated by an $p$-dimensional Gauss function [32], see Eq. 17:

$P(\vec{y} \mid \vec{\xi}) \propto \exp \left\{-\left[\sum \chi^{2}\left(\overrightarrow{\xi_{0}}\right)+\frac{1}{2}\left(\vec{\xi}-\overrightarrow{\xi_{0}}\right)^{T} \vec{H}\left(\vec{\xi}-\overrightarrow{\xi_{0}}\right)\right]\right\}$

The errors of the fit-parameters and the correlation between the fitparameters are determined from the inflection line ( $n$-dimensional ellipse) of the $n$-dimensional Gauss function. These values are represented by a symmetric covariance matrix $C_{i j}$ which can be calculated by inverting the Hesse-Matrix [31] (see Eq. 18). This means that large values in the $2^{\text {nd }}$ derivatives correspond to small errors in the model parameters.

$C_{i j}=\left(\frac{1}{2} H_{i j}\right)^{-1}$

The principal diagonal of the covariance matrix $C_{i j}$ represents the variance of the model parameters. The covariance between two different fit parameters is described by the non-diagonal terms of $C_{i j}$. Microstrain $e$ and dislocation density $\rho$ are not explicit model parameters in our model. That means that the errors of these two parameters must be determined by the classical error propagation using the corresponding terms in the covariance matrix $C_{i j}$ [46]. According to Eq. 15 the 
Table 2

Error propagation for the microstrain $e$ at the end of the isothermal heating step at $650{ }^{\circ} \mathrm{C}$.

\begin{tabular}{|c|c|c|c|c|c|c|c|}
\hline Phase & $E$ & $\sigma_{e \mathrm{~L}}^{2}$ & $\sigma_{e G}^{2}$ & $\sigma_{e \mathrm{~L}, e \mathrm{G}}$ & $r_{e_{1}, e_{\mathrm{G}}}$ & $\sigma_{e}$ & $\frac{\sigma_{e}}{e}$ \\
\hline Martensite & $0.87 \cdot 10^{-3}$ & $0.21 \cdot 10^{-8}$ & $0.07 \cdot 10^{-8}$ & $-0.12 \cdot 10^{-8}$ & -0.925 & $4.3 \cdot 10^{-5}$ & 0.05 \\
\hline Austenite & $2.08 \cdot 10^{-3}$ & $5.93 \cdot 10^{-8}$ & $5.94 \cdot 10^{-8}$ & $-5.93 \cdot 10^{-8}$ & -0.999 & $2.1 \cdot 10^{-5}$ & 0.01 \\
\hline
\end{tabular}

microstrain $e$ is a function of $e_{\mathrm{L}}$ and $e_{\mathrm{G}}$. The variance $\sigma_{e}^{2}$ of the microstrain $e$ is calculated according to Eq. 19 using classical error propagation.

$$
\begin{aligned}
\sigma_{e}^{2}= & \left(\frac{\partial f\left(e_{\mathrm{L}}, e_{\mathrm{G}}\right)}{\partial e_{\mathrm{L}}}\right)^{2} \cdot \sigma_{e_{\mathrm{L}}}^{2}+\left(\frac{\partial f\left(e_{\mathrm{L}}, e_{\mathrm{G}}\right)}{\partial e_{\mathrm{G}}}\right)^{2} \cdot \sigma_{e_{\mathrm{G}}}^{2} \\
& +2\left(\frac{\partial f\left(e_{\mathrm{L}}, e_{\mathrm{G}}\right)}{\partial e_{\mathrm{L}}}\right)\left(\frac{\partial f\left(e_{\mathrm{L}}, e_{\mathrm{G}}\right)}{\partial e_{\mathrm{G}}}\right) \cdot \sigma_{e_{\mathrm{L}}, e_{\mathrm{G}}}
\end{aligned}
$$

The correlation $r_{e \mathrm{~L}}, e \mathrm{G}$ between $e_{\mathrm{L}}$ and $e_{\mathrm{G}}$ is defined according to Eq. 20 using values of the covariance matrix $C_{i j}$ :

$r_{e_{\mathrm{L}}, e_{\mathrm{G}}}:=\frac{\sigma_{e_{\mathrm{L}}, e_{\mathrm{G}}}}{\sigma_{e_{\mathrm{G}}} \cdot \sigma_{e_{L}}}$

In this case the partial derivatives of the function $e$ (Eq. 15) with respect to $e_{\mathrm{L}}$ and $e_{\mathrm{G}}$ are calculated in the vicinity of the minimum of $\Sigma \chi^{2}$. The quantities corresponding to the error estimation of the microstrain are calculated according to Eq. 19 and are presented in Table 2.

The estimated relative error $\frac{\sigma_{e_{1}}}{e_{1}}$ of the microstrain $e_{1}$ is 0.05 . The corresponding relative error of the dislocation density $\rho_{1}$ is 0.1 for martensite. The error of the dislocation density $\rho$ is twice the microstrain $e$ due to the fact that $\rho$ is proportional to $e^{2}$. The correlation.

$r_{1 e \mathrm{~L}, e \mathrm{G}}=-0.925$ approximates almost an anti-correlation behavior between $e_{1 \mathrm{~L}}$ and $e_{1 \mathrm{G}}$.

When analyzing the austenite, the relative error $\frac{\sigma_{e_{2}}}{e_{2}}$ of the microstrain $e_{2}$ is 0.01 and the relative error for $\rho_{2}$ equals 0.02 . The correlation for austenite $r_{2} e \mathrm{~L}, e \mathrm{G}=-0.999$ is even closer to complete anti-correlation between $e_{2 \mathrm{~L}}$ and $e_{2 \mathrm{G}}$ than for martensite.

The approximately $5 \%$ and $1 \%$ relative error of the microstrain (i.e. $10 \%$ and $2 \%$ relative error of the dislocation density) for martensite and austenite seems completely unrealistic and the error estimation is not applicable for these measurements. An extrapolation to an error of one standard deviation fails completely (see also Fig. 10).

Therefore another method is required to calculate the standard deviation of the dislocation densities. To this end histograms obtained from the MCMC simulations are approximated by Gaussian distributions of the dislocation densities $\rho_{1}$ and $\rho_{2}$. Eventually, the standard deviations $\sigma_{\rho 1}$ and $\sigma_{\rho 2}$ of the dislocation densities are calculated from Gaussian distributions. The errors of these quantities are estimated within one standard deviation $\sigma$. The error $\Delta \sigma_{\rho 1}$ for the dislocation density $\rho_{1}$ (martensite) is determined to be $\pm 8 \%$ at $25{ }^{\circ} \mathrm{C}$ (beginning of the heat treatment), $\pm 70 \%$ at $650{ }^{\circ} \mathrm{C}$ (end of isothermal heat treatment) and $\pm 20 \%$ at $25{ }^{\circ} \mathrm{C}$ (after heat treatment). The error $\Delta \sigma_{\rho 2}$ for the dislocation density $\rho_{2}$ (austenite) is extremely uncertain at the beginning of the heat treatment due to a very small volume fraction of austenite below the experimental detection limit. The error $\Delta \sigma_{\rho 2}$ equals $\Delta \sigma_{\rho 2}= \pm$ $50 \%$ at $650{ }^{\circ} \mathrm{C}$ (end of isothermal heat treatment) and $\Delta \sigma_{\rho 2}= \pm 13 \%$ at $25{ }^{\circ} \mathrm{C}$ (after heat treatment).

\section{Conclusion and outlook}

A combination of the Rietveld method (including the fundamental parameter model with the Voigt approach) and a Markov Chain Monte Carlo applied Bayesian approach is presented to evaluate low signal-tonoise ratio diffractograms during a rapid complex solid state phase transformation in a stainless steel leading to phase fractions and dislocation densities not easily captured in conventional in-situ X-ray diffraction experiments. The used highly efficient Markov Chain Monte Carlo algorithm (DREAM) [20] works as global optimization tool and delivers results that are more robust than e.g. results obtained from the Levenberg-Marquardt algorithm. By means of this effective search algorithm it is possible to escape from local minima and thereby reduce biased results obtained from imperfect diffraction data. Such imperfect in-situ X-ray diffraction data of a martensitic stainless steel sample (characterized by a low signal to noise ratio and containing artificial peaks) are analyzed by this approach. The error propagation algorithm with 2nd order Taylor series fails totally for the estimation of the error of the dislocation density for the low signal to noise ratio data used here. In contrast, the used histograms obtained from Markov Chain Monte Carlo method provide reliable results and clear error estimates. It is worth mentioning that the trends of the phase fractions and the dislocation densities of the phases are found to be similar to independent, comparatively high quality X-ray diffraction data reported in [16]. It is expected that the evaluation tool presented in this work has future potential to be applied to robust on-line evaluation of in-situ X-ray diffraction data in particular for fast processes where the diffraction data have a low signal-to-noise ratio.

\section{Data availability}

The raw and processed data required to reproduce these findings are available from the authors.

\section{Declaration of Competing Interest}

The authors declare no competing interest.

\section{References}

[1] S. Raju, K. Sivasubramanian, D. Ramachandran, G. Paneerselvam, A. Banerjee, E. Mohandas, M.P. Antony, Thermal expansion studies on Inconel-600((R)) by high temperature X-ray diffraction, J. Nucl. Mater. 325 (2004) 18-25.

[2] D. Ramachandran, A.M.K. Kirubaharan, A.M. Rabel, P. Kuppusami, Thermal expansion behaviour of Inconel-690 by in situ high temperature X-ray diffraction, Mater. Sci. Forum 830-831 (2015) 367-370.

[3] R. Pederson, O. Babushkin, F. Skystedt, R. Warren, Use of high temperature X-ray diffractometry to study phase transitions and thermal expansion properties in Ti6Al-4V, Mater. Sci. Technol. 19 (2003) 1533-1538.

[4] A.K. Chawla, R. Chandra, Synthesis and structural characterization of nanostructured copper, J. Nanopart. Res. 11 (2009) 297-302.

[5] A. Bojack, L. Zhao, P.F. Morris, J. Sietsma, In-situ determination of austenite and martensite formation in 13Cr6Ni2Mo supermartensitic stainless steel, Mater. Charact. 71 (2012) 77-86.

[6] H. Kotan, K.A. Darling, Phase transformation and grain growth behaviour of a nanocrystalline 18/7 stainless steel, Mater. Sci. Eng. A 686 (2017) 168-175.

[7] D. Garipoli, P. Bergese, E. Bontempi, M. Minicucci, A. Di Cicco, L.E. Depero, High temperature X-ray diffraction investigation of an aluminium-silicon-corundum system, Z. Kristallogr. Suppl. 26 (2007) 369-374.

[8] K.A. Atmane, F. Zighem, Y. Soumare, M. Ibrahim, R. Boubekri, T. Maurer, J. Margueritat, J.-Y. Piquemal, F. Ott, G. Chaboussant, F. Schoenstein, N. Jouini, G. Viau, High temperature structural and magnetic properties of cobalt nanorods, J. Solid State Chem. 197 (2013) 297-303.

[9] C.-Y. Hsu, C.-C. Juan, W.-R. Wang, T.-S. Sheu, J.-W. Yeh, S.-K. Chen, On the superior hot hardness and softening resistance of $\mathrm{AlCoCr}_{\mathrm{x}} \mathrm{FeMo}_{0.5} \mathrm{Ni}$ high-entropy alloys, Mater. Sci. Eng. A 528 (2011) 3581-3588.

[10] A. Teresiak, A. Gebert, M. Savyiak, M. Uhlemann, Ch. Mickel, N. Mattern, In situ high temperature XRD studies of the thermal behavior of the rapidly quenched Mg77Ni18Y5 alloy under hydrogen, J. Alloys Compd. 398 (2005) 156-164.

[11] J. Zhang, T. Lu, X. Chang, S. Jiang, N. Wei, J. Qi, Yield strength of transparent $\mathrm{MgAl} 2 \mathrm{O} 4$ Nano-ceramic at high pressure and temperature, Nanoscale Res. Lett. 5 (2010) 1329-1332.

[12] R.W. Cheary, A.A. Coelho, J.P. Cline, Fundamental parameters line profile fitting in laboratory diffractometers, J. Res. Natl. Inst. Stand. Technol. 109 (2004) 1-25.

[13] TOPAS 4.2 User Manual, Bruker AXS GmbH, Germany, 2009. 
[14] D. Balzar and H. Ledbetter, Accurate modeling of size and strain broadening in the Rietveld refinement: the "double-Voigt" approach, advances in X-ray analysis 38 plenum press, N. Y. (1995) 397-404.

[15] M. Wießner, S. Kleber, A. Kulmburg, In-situ investigation during tempering of a high speed steel with X-ray diffraction, Part. Part. Syst. Charact. 64 (2006) 407-417.

[16] M. Wiessner, E. Gamsjäger, S. van der Zwaag, P. Angerer, Effect of reverted austenite on tensile and impact strength in a martensitic stainless steel-an in-situ X-ray diffraction study, Mater. Sci. Eng. A 682 (2017) 117-125.

[17] T. Shintani, Y. Murata, Evaluation of the dislocation density and dislocation character in cold rolled type 304 steel determined by profile analysis of X-ray diffraction, Acta Mater. 59 (2011) 4314-4322.

[18] J. Pešička, R. Kužel, A. Dronhofer, G. Eggeler, The evolution of dislocation density during heat treatment and creep of tempered martensite ferritic steels, Acta Mater. 51 (2003) 4847-4862.

[19] L.B. McCusker, R.B. Von Dreele, D.E. Cox, D. Louër, P. Scardi, Rietveld refinement guidelines, J. Appl. Crystallogr. 32 (1999) 36-50.

[20] J.A. Vrugt, C.J.F. Ter Braak, DREAM(D): an adaptive Markov chain Monte Carlo simulation algorithm to solve discrete, noncontinuous, and combinatorial posterior parameter estimation problems, Hydrol. Earth Syst. Sci. 15 (2011) 3701-3713.

[21] C.J.F. Ter Braak, A Markov chain Monte Carlo version of the genetic algorithm differential evolution: easy Bayesian computing for real parameter space, Stat. Comput. 16 (2006) 239-249.

[22] J.A. Vrugt, C.J.F. ter Braak, C.G.H. Diks, B.A. Robinson, J.M. Hyman, D. Higdon, Accelerating Markov chain Monte Carlo simulation by differential evolution with self-adaptive randomized subspace sampling, Int. J. Nonlinear Sci. Num. Simul. 10 (2009) 273-290.

[23] M. Wießner, Hochtemperatur-Phasenanalyse am Beispiel martensitischer Edelstähle, epubli, 2010.

[24] M. Wiessner, P. Angerer, Bayesian approach applied to the Rietveld method, J. Appl. Crystallogr. 47 (2014) 1819-1825.

[25] J.E. Lesniewski, S.M. Disseler, D.J. Quintana, P.A. Kienzle, W.D. Ratcliff, Bayesian method for the analysis of diffraction patterns using BLAND, J. Appl. Crystallogr. 49 (2016) 2201-2209.

[26] E. Kreyszig, Advanced Engineering Mathematics, 7th ed., John Wiley \& Sons, 1993.

[27] W.M. Bolstad, Introduction to Bayesian Statistics, John Wiley \& Sons, 2005.

[28] D.J.C. MacKay, Information Theory, Inference, and Learning Algorithms, Version 7.2, Cambridge University Press, 2005.

[29] J. Wolberg, Data Analysis Using the Method of Least Squares, Springer, 2006.
[30] Bradley E. Huitema, The Analysis of Covariance and Alternatives: Statistical Methods for Experiments, Quasi-Experiments, and Single-Case Studies, Second edition, Wiley and Sons, 2011.

[31] K. Levenberg, A method for the solution of certain problems in least squares, Q. Appl. Math. 2 (1944) 164-168.

[32] D. Marquardt, An algorithm for least-squares estimation of nonlinear parameters, SIAM J. Appl. Math. 11 (1963) 431-441.

[33] D. Sivia, J. Skilling, Data Analysis: A Bayesian Tutorial, Oxford University Press, 2006.

[34] N. Metropolis, A. Rosenbluth, M. Rosenbluth, A. Teller, E. Teller, Equation of state calculations by fast computing machines, J. Chem. Phys. 21 (1953) 1087-1092.

[35] W.K. Hastings, Monte Carlo sampling methods using Markov chains and their applications, Biometrika 57 (1970) 97-109.

[36] N404 Stainless Steel Bohler N404. https://www.bohler-edelstahl.com/en/produ cts/n404/, 2020 (Accessed 25.10.2020).

[37] J.I. Langford, R. Delhez, Th.H. de Keijser, E.J. Mittemeijer, Profile analysis for microcrystalline properties by the Fourier and other methods, Aust. J. Phys. 41 (1988) 173-187.

[38] D.C. Hurley, D. Balzar, P.T. Purtscher, K.W. Hollman, Nonlinear ultrasonic parameter in quenched martensitic steels, J. Appl. Phys. 83 (1998) 4584-4588.

[39] F. Richter, Die physikalischen Eigenschaften der Stähle: Das 100 - Stähle Programm "The Physical Properties of Steels, The 100 Steels Programme". https ://www.tugraz.at/fileadmin/user_upload/Institute/IEP/Thermophysics_Group/Fi les/Staehle-Richter.pdf, 2020 (Accessed 25.10.2020).

[40] Standard Reference Material 660a Lanthanum Hexaboride Powder Diffraction, National Institute of Standards \& Technology, Gaithersburg, Maryland, 2000.

[41] J. Hill, I.C. Madsen, The effect of profile step counting time on the determination of crystal structure parameters by X-ray Rietveld analysis, J. Appl. Crystallogr. 17 (1984) 297-306.

[42] A. Gagin, I. Levin, Accounting for unknown systematic errors in Rietveld refinements: a Bayesian statistics approach, J. Appl. Crystallogr. 48 (2015) 1201-1211.

[43] E. Baharie, G.S. Pawley, Counting statistics and powder diffraction scan refinements, J. Appl. Crystallogr. 16 (1983) 404-406.

[44] T. Ida, M. Ando, H. Toraya, Extended pseudo-Voigt function for approximating the Voigt profile, J. Appl. Crystallogr. 16 (1983) 404-406.

[45] H.O. Di Rocco, A. Cruzado, The Voigt profile as a sum of a Gaussian and a Lorentzian functions, when the weight coefficient depends only on the widths ratio, Acta Phys. Pol. A 33 (2000) 1311-1316.

[46] H.H. Ku, Notes on the use of propagation of error formulas, J. Res. Natl. Bur. Stand. 70C (1966) 263-273. 